\title{
A microcontroller-based simulation of dural venous sinus injury for neurosurgical training
}

\author{
*Daniel R. Cleary, MD, PhD, ${ }^{1,2}$ Dominic A. Siler, MD, PhD, ${ }^{1}$ Nathaniel Whitney, MD, MS, ${ }^{1}$ and \\ Nathan R. Selden, MD, PhD' \\ 'Department of Neurological Surgery, Oregon Health \& Science University, Portland, Oregon; and 2Department of Neurological \\ Surgery, University of California, San Diego, California
}

\begin{abstract}
OBJECTIVE Surgical simulation has the potential to supplement and enhance traditional resident training. However, the high cost of equipment and limited number of available scenarios have inhibited wider integration of simulation in neurosurgical education. In this study the authors provide initial validation of a novel, low-cost simulation platform that recreates the stress of surgery using a combination of hands-on, model-based, and computer elements. Trainee skill was quantified using multiple time and performance measures. The simulation was initially validated using trainees at the start of their intern year.
\end{abstract}

METHODS The simulation recreates intraoperative superior sagittal sinus injury complicated by air embolism. The simulator model consists of 2 components: a reusable base and a disposable craniotomy pack. The simulator software is flexible and modular to allow adjustments in difficulty or the creation of entirely new clinical scenarios. The reusable simulator base incorporates a powerful microcomputer and multiple sensors and actuators to provide continuous feedback to the software controller, which in turn adjusts both the screen output and physical elements of the model. The disposable craniotomy pack incorporates 3D-printed sections of model skull and brain, as well as artificial dura that incorporates a model sagittal sinus.

RESULTS Twelve participants at the 2015 Western Region Society of Neurological Surgeons postgraduate year 1 resident course ("boot camp") provided informed consent and enrolled in a study testing the prototype device. Each trainee was required to successfully create a bilateral parasagittal craniotomy, repair a dural sinus tear, and recognize and correct an air embolus. Participant stress was measured using a heart rate wrist monitor. After participation, each resident completed a 13-question categorical survey.

CONCLUSIONS All trainee participants experienced tachycardia during the simulation, although the point in the simulation at which they experienced tachycardia varied. Survey results indicated that participants agreed the simulation was realistic, created stress, and was a useful tool in training neurosurgical residents. This simulator represents a novel, lowcost approach for hands-on training that effectively teaches and tests residents without risk of patient injury.

https://thejns.org/doi/abs/10.3171/2016.12.JNS162165

KEY WORDS simulation; hemorrhage; sagittal sinus; neurological surgery; residency training

$\mathrm{N}$ EUROSURGICAL intraoperative emergencies require rapid clinical decision making under conditions of high stress. Nationally mandated work-hour restrictions may reduce access to operative experience crucial to encountering pivotal, rare intraoperative emergencies. ${ }^{8,22}$ Simultaneously, public and regulatory scrutiny has increased pressure on supervising physicians to directly manage any irregular clinical and operative occurrences. ${ }^{17}$ In combination, these factors pose significant risk that practitioners will first encounter and manage serious adverse events without experience or direct support.

In 2009, postgraduate year 1 (PGY1) neurological surgery residents attended a Society of Neurological Surgeons (SNS) pilot "boot camp" course designed in part to enhance their familiarity with and safety performing neurosurgical procedures and basic operative skills prior to practicing them in the live clinical environment. ${ }^{24,25}$ Simulation training early in residency is effective in improving

ABBREVIATIONS bpm = beats per minute; HR = heart rate; OHSU = Oregon Health \& Science University; PGY1 = postgraduate year $1 ;$ SNS = Society of Neurological Surgeons.

SUBMITTED August 24, 2016. ACCEPTED December 5, 2016.

INCLUDE WHEN CITING Published online June 2, 2017; DOI: 10.3171/2016.12.JNS162165.

* Drs. Cleary and Siler contributed equally to this work. 
residents' confidence in and proficiency with basic technical skills, as well as knowledge retention. ${ }^{3,9,23}$

Neurosurgery program directors embrace simulation as a supplement to traditional educational approaches for the improvement of clinical outcomes. ${ }^{10}$ Simulation may enhance practice-based skills performance improvement. . $^{1,4,6,13,16,20,26}$ While simulation appears to be a valuable complement to traditional surgical training, more studies are needed to define what simulation modalities and methods are most useful. ${ }^{10,29}$

Cadaver-based simulation is a long-established educational method in neurological surgery, more recently supplemented with model and computer-based simulations. ${ }^{17,11,12,14,18}$ Widespread adoption of neurosurgical simulators has been limited by high cost and a limited availability of easily accessible, dynamic simulation models for resident education.

In this paper we describe a neurosurgical simulation physical model with adaptive computational components. The simulation utilizes an anatomically correct physical model supplemented with sensors, actuators, and a combination microcontroller/microcomputer. The simulation exercise combines technical tasks, such as drilling and suturing, with acute intraoperative complication management and decision making related to both sagittal sinus bleeding and venous air embolism. We also present data collected in an initial trial of the simulator exercise as part of the 2015 SNS Western Region Boot Camp in Portland, Oregon.

\section{Methods \\ Overview}

A novel venous sinus injury and venous air embolus surgical simulation was tested at the SNS Western Region PGY1 boot camp course held at Oregon Health \& Science University (OHSU) on July 11, 2015. Human subject participation was approved by the OHSU Institutional Review Board. Twelve individuals (11 trainees and 1 attending physician) provided informed consent and participated in this prototype trial.

The simulation goals were to experience a surgical emergency, manage a sagittal sinus injury with severe bleeding, and manage venous air embolism. The simulation used a combination of hands-on human anatomy models, sensors and actuators within the model, and an underlying software base that controls the simulation, supports data collection, and displays simulated vital signs. Additional equipment required for this simulation included a surgical table or stretcher with Trendelenburg position capability, surgical drills, suture material, and standard craniotomy surgical instruments. OHSU neurosurgical faculty acted as proctors and provided feedback about the device and trainee performance.

\section{Hardware and Software Platform}

The system is built using UDOO (www.udoo.org; SECO USA Inc.), a combination microcontroller and microcomputer. UDOO contains all the components of a fully functioning computer, including a $1-\mathrm{GHz}$ central processing unit, independent graphics processing chips, wireless mod- ule, and audio output. UDOO is accessed with a keyboard, monitor, and speakers, but can also be programmed to function without user input. UDOO also has an integrated microcontroller platform that facilitates device design and prototyping. The microcontroller component of the system allows incorporation of environmental sensors (temperature, light, angle, and motion) and actuators (motors, physical switches, and light-emanating diodes) into the core program. Although only a few sensors and actuators were used in this simulation, the platform has additional functionality that will allow additional simulation variable expansion for new scenarios and models.

Two separate computer programs were written: one to run on the microcomputer, and one to run on the microcontroller. The software running on the microcomputerincluding the graphical user interface, the simulation plan, and a module for interfacing with the microcontrollerwas written in the computer language Python (www. python.org) using the custom interface library PyGame (www.pygame.org). The software displays the simulated patient's vital signs in real time, including heart rate (HR), respiratory rate, blood oxygenation, blood pressure, and central venous pressure. The program features were designed as modules, allowing for easy interchange of features for other simulations. When initiated, the program displays the vital signs in a continuous stream, and then follows the outlined sequence of events (Fig. 1), which included taking information from the user, displaying the start screen, updating and changing the vital signs, and displaying help screens. In addition to displaying data on the screen, the program takes metrics of individual performance based on the sensor data and time.

The microcontroller software is simple, containing only core components for reading sensors, running actuators, and receiving instructions and relaying data to and from the main program. The sensors used in this simulation were an accelerometer for determining the position of the head (Trendelenburg vs reverse Trendelenburg), a flowmeter for quantifying blood loss during the simulation, and the internal clock for measuring time elapsed. The actuator used during this simulation was a flow controller for starting and stopping the flow of simulated blood during the simulation. This software component was built using Processing (www.processing.org), a simple programming language used with microcontrollers. The microcontroller and the microcomputer communicate via the internal UDOO hardware.

\section{Physical Model}

The model was a customized mannequin head (Giell Supply) with realistic external anatomical landmarks. For use with the simulator, the head was shaved and a coronal incision was made in the synthetic skin. The underlying Styrofoam was removed, and the compartment was sealed against water using a plastic resin. The UDOO board and accompanying sensors and actuators were installed in the posterior compartment and a disposable craniotomy pack installed directly under the incision. The completed simulator unit was then attached to a surgical table using a Mayfield head immobilization device (Integra Life Sciences). 


\section{Simulation Course}

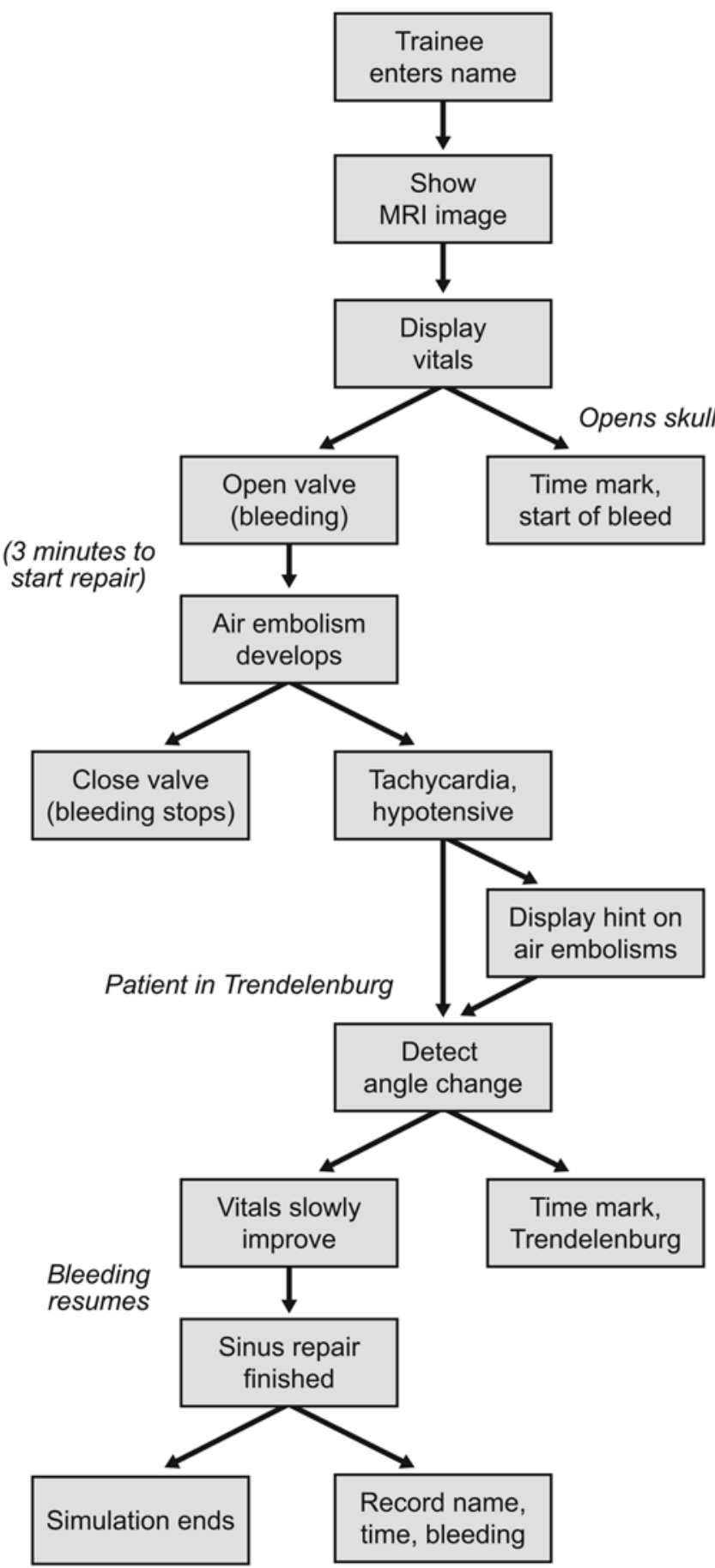

FIG. 1. Design flowchart of the simulation course (see Methods).

\section{Craniotomy Pack}

The craniotomy pack for the sagittal sinus injury-venous air embolism simulation was created using 4 components. At the base of the pack was a 3D-printed segment of brain including the medial frontal convexity and the interhemispheric fissure. Overlying the brain was a layer of Tyvek fabric (DuPont), a lightweight and strong synthetic fabric that has similar thickness and tactile quality to human dura. To replicate the superior sagittal sinus, a segment of soft, thin-walled silicone tubing was connected to a double-layered section of the Tyvek using silicone adhesive. The Tyvek mimicked the physical aspects of the sinus, and the tubing connection allowed a continuous flow of liquid into the sinus. To simulate the tear in the sinus, an 8 -mm ovoid hole was cut into the superior surface of the Tyvek. This hole was attached to the 3D-printed skull using hot melt adhesive, preventing simulated bleeding until the moment of bone flap removal. The skull was printed using acrylonitrile butadiene styrene plastic and designed with thicker outer and inner layers as well as a trabeculated internal structure, replicating the structure of cortical and cancellous bone for the drilling portion of the simulation. The skull was attached and drill sites premarked such that the hole in the sinus would be exposed at the moment of bone flap removal.

Each completed craniotomy pack was designed for disposable single use. Prior to each new simulation, a new craniotomy pack was installed and secured using hot melt adhesive. The silicone tubing from the simulated sagittal sinus was then connected to a reservoir of water slightly thickened with corn syrup and colored with blue food coloring dye. Simulated bleeding flow rate was measured and controlled using a small flowmeter and a flow-switch controller, both connected to the microcontroller unit.

\section{Simulation Course}

The neurosurgical simulation was designed to replicate superior sagittal sinus injury during a craniotomy, incorporating necessary technical maneuvers and decision making to manage rapid bleeding and venous air embolism. A simulation proctor assists with setup before each trainee begins, responds to queries during the simulation, and debriefs the trainee after the simulation is complete. The simulation course is outlined in Fig. 1. The trainee dons a surgical gown, gloves, and eye protection. The proctor then briefly informs the trainee that he or she will participate in a surgical simulation and to follow the onscreen instructions. A screen initially displays a coronal MR image of a 5-cm, well-circumscribed parasagittal frontal mass, as well as basic instructions for localizing an appropriate craniotomy.

Surgical instruments, suction, gauze, and a high-speed drill with perforator and craniotome attachments are available. The trainee is required to fashion a right-biased bilateral frontal craniotomy with 4 bur holes that crosses the midline. When the artificial bone flap is removed, sagittal sinus bleeding begins at approximately $5 \mathrm{ml} / \mathrm{sec}$ $(300 \mathrm{ml} / \mathrm{min})$. The flowmeter incorporated into the tubing quantifies total blood loss. The trainee is expected to use basic immediate interventions to reduce the bleeding rate, such as manual compression. They must then devise and carry out a definitive sinus repair. If after 2 minutes bleeding continues at a high rate, on-screen instructions appear describing how to repair the sinus injury with a small dural rotational patch (Fig. 2).

Four minutes after the start of sinus bleeding, the flowswitch controller stops blood flow while vital signs displayed on the patient monitor and represented by tones 


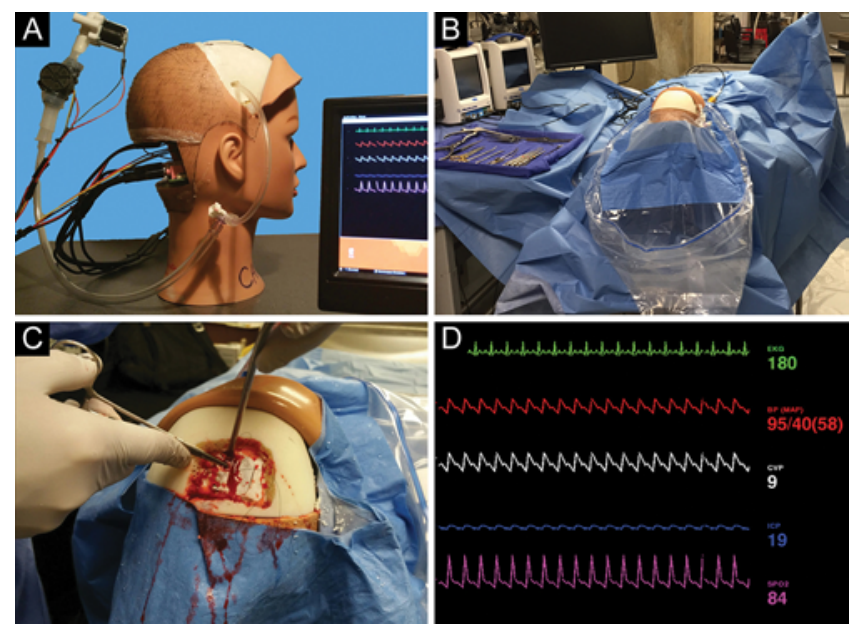

FIG. 2. Photographs of simulation materials. A: Completed device prototype, with all components mounted inside the base head model, showing the flow controller, flowmeter, and associated tubing. B: Simulation equipment setup. Additional equipment included operating drapes, towels, a craniotomy drill, standard surgical tools, and suture material. C: A trainee undertaking dural venous sinus repair during the simulation. D: Changes in vital signs with development of a venous air embolus, including tachycardia, hypotension, and oxygen desaturation, are displayed on a clinical monitor during the simulation. Figure is available in color online only.

indicate tachycardia, hypotension, and hypoxia, simulating a venous air embolism. If the trainee did not notice this change in clinical status after 1 minute, the proctor prompted them to respond. Appropriate interventions include flooding the surgical field with irrigation, placing the patient in the Trendelenburg position (tracked by an accelerometer in the surgical table), and occluding the site of sinus injury. Appropriate treatment results in improving vital signs and resumption of sinus bleeding, at which time the trainee should complete their sinus repair. The simulation ends when bleeding is fully stopped and vital signs have normalized, or when time expires. The proctor then debriefs the trainee and explains the pathophysiology and management of dural sinus tear and venous air embolism.

\section{Testing User Performance With the Prototype Device}

During simulation, each participant wore a wristmounted continuous HR monitor. Baseline HR was measured in the simulation testing room during preparation. Comparisons were made based on the mean HR and periods of peak HR. Tachycardia was defined as HR greater than 100 beats per minute (bpm). Comparisons of mean HR within participants were made using a paired t-test, with significance defined as $p<0.05$. Comparisons of relative time spent tachycardic within different segments of the simulation were made using 1-way ANOVA.

In addition, each participant completed a nonvalidated, IRB-approved, immediate post-simulation experience survey instrument. The survey consisted of 13 categorical questions written to assess the accuracy of the simulator, ease of use, and degree of "immersion" the participant felt during the simulation. The available responses were on a Likert-like scale, ranging from "strongly disagree" to "strongly agree."

\section{Results \\ Heart Rate Tracings}

Eleven trainees and 1 attending physician participated in the study. Data from the trainees are presented separately from those of the attending physician. HR tracings were recorded from participants as a surrogate measure of anxiety and the autonomic response to stress. Figure 3A shows an example of a trainee HR response during the simulation. Variation in trainee HR occurred principally at specific stress points during the stimulation, including occurrence of the venous sinus injury during craniotomy (typically tachycardia) and patient vital sign changes with venous air embolus (typically tachycardia or bradycardia followed by tachycardia). By comparison, the attending physician (Fig. 3B) showed only a mild rise in HR after venous sinus injury but no tachycardia. Mean HR among trainees was $24 \mathrm{bpm}$ higher than that of the single attending physician participant (Fig. 3C). Overall, the trainee participants experienced a statistically significant rise in HR during the simulation (Fig. 3D).

To analyze trainee data, HR tracings were divided into 4 simulation stages: drilling, venous sinus injury, air embolism, and suturing. We quantified the relative amount of time within each segment that each participant was tachycardic (HR $\geq 100 \mathrm{bpm})$. During simulation preparation, 27\% of participants were tachycardic at baseline. All trainees showed tachycardia during at least 1 stage of the simulation, although the specific point at which they became tachycardic varied. Within any given segment, $63 \%$ of trainees were tachycardic. The mean percentage of time that trainees spent in tachycardia was not significantly different between segments.

\section{Simulation Survey}

The results of the post-simulation survey are shown in Fig. 4. The majority of participants agreed that the simulation was realistic and created a sense of urgency during the simulated complications (e.g., "Great tool for managing a rare but serious complication" and "Very good practice to allow clinical decision making intraoperatively"). Additionally, only a small minority of participants reported distraction by nonsimulation-related surroundings and the majority of participants were thus able to focus on core simulation tasks. All participants felt the simulation would be a useful tool in neurosurgical training.

\section{Discussion}

Simulation is an effective adjunct to traditional surgical training, but it is in the early stages of integration into neurosurgical education. ${ }^{2}$ One of the major limitations of widespread inclusion of simulation in education has been cost. Expensive and delicate simulation equipment may restrict availability and repeat use..$^{5,11}$ In this study, we mitigated this limitation by creating an inexpensive reusable base model with a low-cost disposable component for individual trainees. The prototype uses off-the-shelf com- 

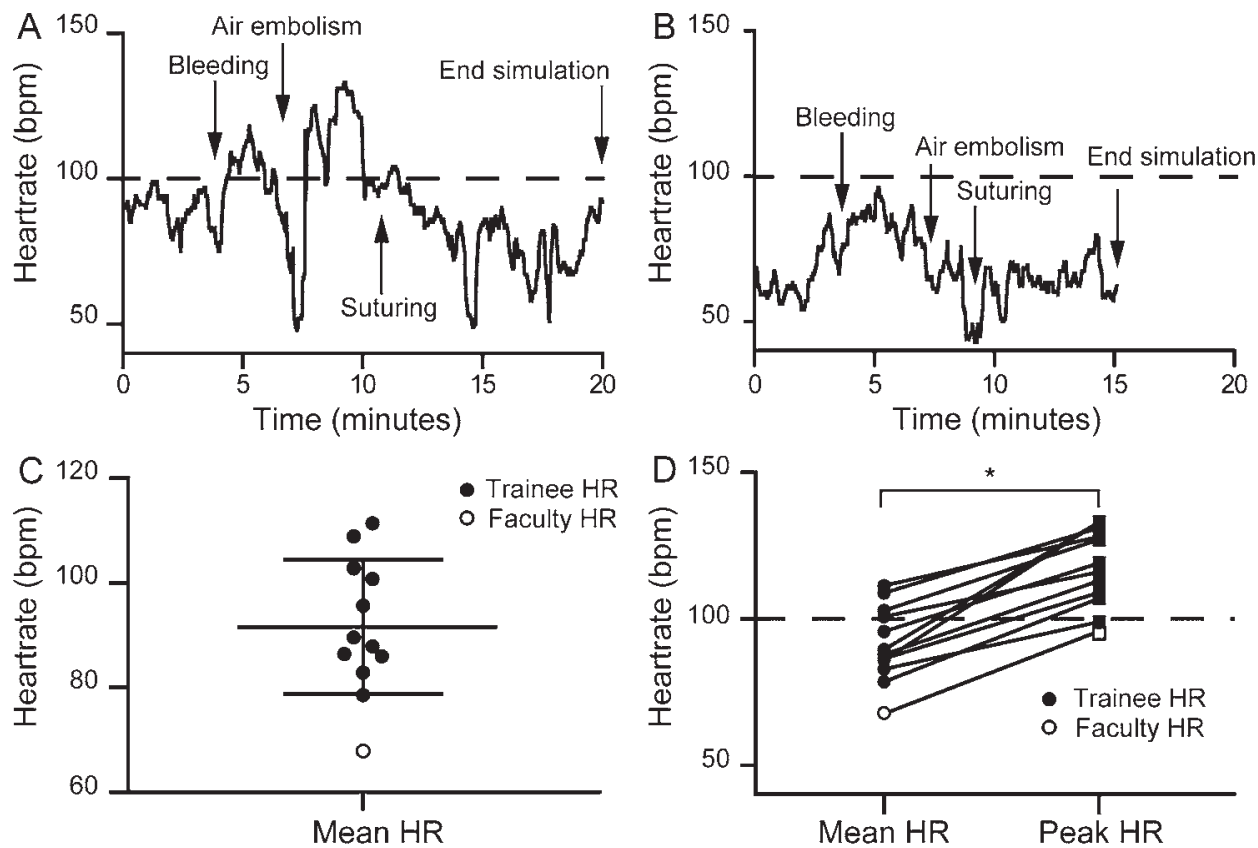

FIG. 3. A: A neurosurgical trainee (Trainee HR) demonstrates periodic tachycardia in association with the events of the simulation. B: An attending physician (Faculty HR) does not demonstrate tachycardia during the simulation, although there are small variations of HR during simulation events. C: Mean HR of both trainee and faculty study participants. D: Peak HR compared with mean HR for trainee and faculty study participants $(n=12) .{ }^{*} p<0.05$.

ponents and was programmed using open source rather than proprietary programming languages, thus avoiding licensing costs. The total cost of the assembled prototype system is less than $\$ 300$ in parts and materials. For each trainee, the disposable component costs less than $\$ 10$ with a manual assembly time of less than 15 minutes.

Most surgical simulators fill 1 of 2 broad categories: anatomical/physical models and computational simulation with haptic feedback. ${ }^{19}$ Physical models (including cadaveric dissection) provide the most realistic anatomical approaches and tactile feedback, but unlike the human body are often static and unresponsive. Computational models, conversely, can adapt and change as the simulation progresses, but even with modern haptic methods lack realism. In this study we have combined the advantages of physical models and computer-based simulation by using a microcomputer and microcontroller. The integrated sensors and actuators change the system based on trainee responses, yet the responses are not confined to those prescribed by the program; that is, trainees were open to use any method of their choosing to stop blood flow. In addition, the physical model in conjunction with surgical gloves, gown, instruments, and functioning drills replicated the tactile and immersive surgical experience.

Stress may facilitate or inhibit learning, depending on the context and learning situation. ${ }^{15,27}$ While it is tempting to try to provide a "stress-free" environment for learning, surgical emergencies demand effective focus and coping skills under stress. Venous sinus injury with massive bleeding and air embolus are potentially catastrophic intraoperative complications that require rapid diagnosis and then the simultaneous exercise of definitive cognitive and manual skills for successful management. Although their occurrence in cranial neurosurgical practice is inevitable, these events are rare. Current trends in North American residency training have increased pressure for faculty to directly manage serious complications as well as potentially reduced trainees' exposure to them given duty-hour restrictions. Realistic simulation provides an alternative methodology for training residents to manage rarely encountered events with crucial skills that profoundly affect patient outcome.

The sagittal sinus injury simulation does appear to generate stress, based on our measurement of tachycardia in all participants during some phase of the simulation. Post-simulation survey results indicate that trainees experience the simulation as realistic and effective. In contrast, a single experienced attending physician participant did not display tachycardia at any time. Simulation during cardiac life-support training has been validated as improving patient outcomes in the live clinical setting. ${ }^{21,28}$ Whether exposure to simulated surgical emergencies similarly improves clinical outcomes is less well understood. Although the impact on clinical performance at unpredictable future dates, with reliable clinical outcome indicators, is complex and difficult, future studies of surgical complication management could quantify degree of improvement using later simulation retesting.

Neurological surgery residency program directors indicate that simulation is an appropriate supplementary training modality that may improve clinical outcomes, ${ }^{10}$ but more studies and a broader range of models are needed to validate this approach. The system we present here will be useful in testing this approach and it may be reconfigured 


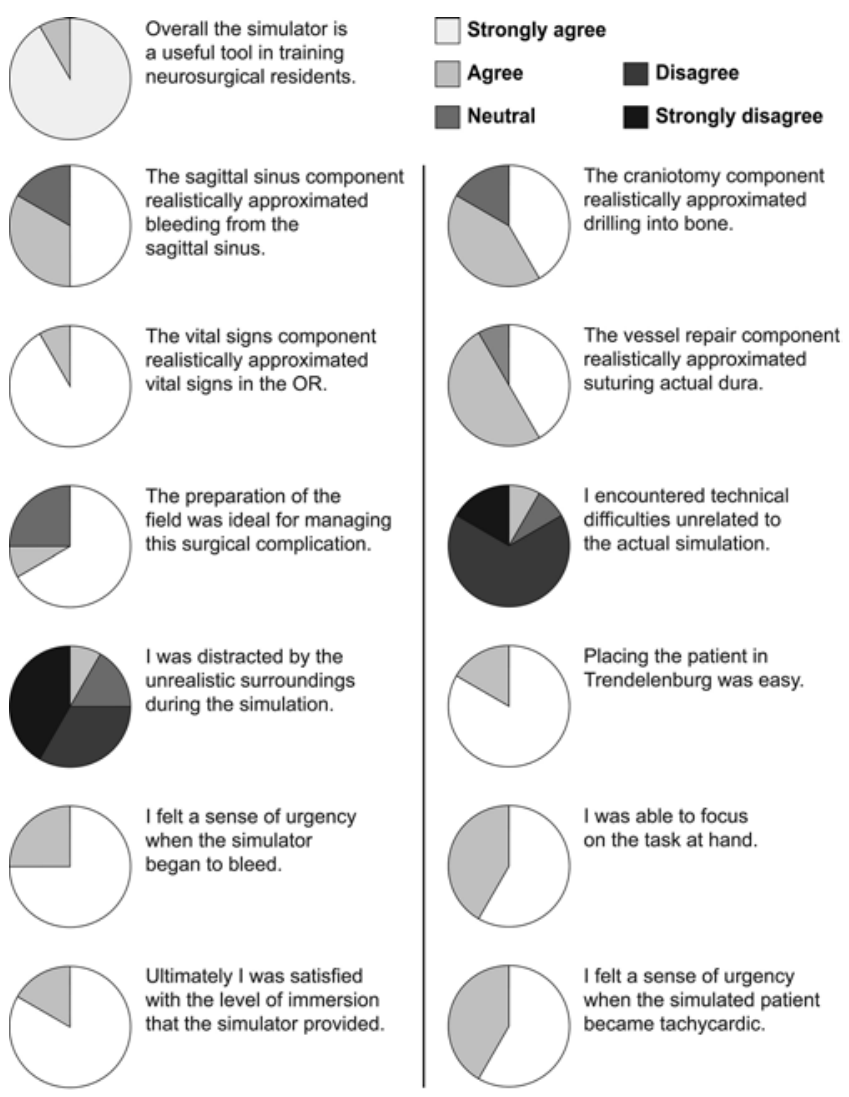

FIG. 4. Portion of categorical responses to survey questions regarding experience on the simulation. Responses are organized by question. OR = operating room.

to simulate other serious intraoperative complications (e.g., cerebral edema, major intracranial arterial injury, etc.).

This study was limited by the small number of subjects studied in the pilot simulation, inability to retest the same subjects after an interval, and inability to test the effect on performance in the live clinical environment. In addition, the post-simulation survey used has not been validated. Nevertheless, we believe the curricular and technical components of this simulation are novel and provide a strong basis for further work in simulating rare, catastrophic intraoperative complications.

\section{Conclusions}

We describe a low-cost, versatile surgical simulation that incorporates the advantages of both physical simulation models and computer control to replicate sagittal sinus injury, a high-stress and high-consequence intraoperative complication. Eleven neurosurgical PGY1 residents and 1 attending physician participated in the simulation pilot testing. All participants showed some degree of stress during participation, based on monitored tachycardia. All participants reported that the simulation was a positive and beneficial learning experience. This prototype represents a new approach to low-cost simulation of rare, catastrophic intraoperative events with the potential to improve neurosurgical training.

\section{Acknowledgments}

We would like to thank Shirley McCartney, $\mathrm{PhD}$, for editorial assistance, and Andy Rekito, MS, for assistance with figures. The authors received financial support from the Cameron Family Foundation Fund, Department of Neurological Surgery, OHSU. The Cameron Family Foundation funded the development and fabrication of the simulator model components. Stryker funded the SNS Western Region PGY1 boot camp course at which the simulator was piloted and data collected, but did not provide any funding for the project described in this paper.

\section{References}

1. Aggarwal R, Black SA, Hance JR, Darzi A, Cheshire NJ: Virtual reality simulation training can improve inexperienced surgeons' endovascular skills. Eur J Vasc Endovasc Surg 31:588-593, 2006

2. Alaraj A, Charbel FT, Birk D, Tobin M, Luciano C, Banerjee $\mathrm{PP}$, et al: Role of cranial and spinal virtual and augmented reality simulation using immersive touch modules in neurosurgical training. Neurosurgery 72 (Suppl 1):115-123, 2013 (Erratum in Neurosurgery 73:E913, 2013)

3. Banerjee PP, Luciano CJ, Lemole GM Jr, Charbel FT, Oh MY: Accuracy of ventriculostomy catheter placement using a head- and hand-tracked high-resolution virtual reality simulator with haptic feedback. J Neurosurg 107:515-521, 2007

4. Chaer RA, Derubertis BG, Lin SC, Bush HL, Karwowski JK, Birk D, et al: Simulation improves resident performance in catheter-based intervention: results of a randomized, controlled study. Ann Surg 244:343-352, 2006

5. Danzer E, Dumon K, Kolb G, Pray L, Selvan B, Resnick AS, et al: What is the cost associated with the implementation and maintenance of an ACS/APDS-based surgical skills curriculum? J Surg Educ 68:519-525, 2011

6. Dawson DL, Meyer J, Lee ES, Pevec WC: Training with simulation improves residents' endovascular procedure skills. J Vasc Surg 45:149-154, 2007

7. Delorme S, Laroche D, DiRaddo R, Del Maestro RF: NeuroTouch: a physics-based virtual simulator for cranial microneurosurgery training. Neurosurgery 71 (1 Suppl Operative):32-42, 2012

8. Dumont TM, Rughani AI, Penar PL, Horgan MA, Tranmer BI, Jewell RP: Increased rate of complications on a neurological surgery service after implementation of the Accreditation Council for Graduate Medical Education work-hour restriction. J Neurosurg 116:483-486, 2012

9. Fann JI, Calhoon JH, Carpenter AJ, Merrill WH, Brown JW, Poston RS, et al: Simulation in coronary artery anastomosis early in cardiothoracic surgical residency training: the Boot Camp experience. J Thorac Cardiovasc Surg 139:12751281,2010

10. Ganju A, Aoun SG, Daou MR, El Ahmadieh TY, Chang A, Wang L, et al: The role of simulation in neurosurgical education: a survey of 99 United States neurosurgery program directors. World Neurosurg 80:e1-e8, 2013

11. Gasco J, Holbrook TJ, Patel A, Smith A, Paulson D, Muns A, et al: Neurosurgery simulation in residency training: feasibility, cost, and educational benefit. Neurosurgery 73 (Suppl 1):39-45, 2013

12. Ghobrial GM, Anderson PA, Chitale R, Campbell PG, Lobel DA, Harrop J: Simulated spinal cerebrospinal fluid leak repair: an educational model with didactic and technical components. Neurosurgery 73 (Suppl 1):111-115, 2013

13. Grober ED, Hamstra SJ, Wanzel KR, Reznick RK, Matsumoto ED, Sidhu RS, et al: The educational impact of bench model fidelity on the acquisition of technical skill: the use of clinically relevant outcome measures. Ann Surg 240:374381,2004

14. Hooten KG, Lister JR, Lombard G, Lizdas DE, Lampotang 
S, Rajon DA, et al: Mixed reality ventriculostomy simulation: experience in neurosurgical residency. Neurosurgery 10 (Suppl 4):576-581, 2014

15. Joëls M, Pu Z, Wiegert O, Oitzl MS, Krugers HJ: Learning under stress: how does it work? Trends Cogn Sci 10:152158,2006

16. Kahol K, Satava RM, Ferrara J, Smith ML: Effect of shortterm pretrial practice on surgical proficiency in simulated environments: a randomized trial of the "preoperative warmup" effect. J Am Coll Surg 208:255-268, 2009

17. Kohn LT, Corrigan JM, Donaldson MS: To Err is Human: Building a Safer Health System. Washington, DC: National Academies Press, 2000, Vol 6

18. Lobel DA, Elder JB, Schirmer CM, Bowyer MW, Rezai AR: A novel craniotomy simulator provides a validated method to enhance education in the management of traumatic brain injury. Neurosurgery 73 (Suppl 1):57-65, 2013

19. Malone HR, Syed ON, Downes MS, D'Ambrosio AL, Quest DO, Kaiser MG: Simulation in neurosurgery: a review of computer-based simulation environments and their surgical applications. Neurosurgery 67:1105-1116, 2010

20. Matsumoto ED, Hamstra SJ, Radomski SB, Cusimano MD: The effect of bench model fidelity on endourological skills: a randomized controlled study. J Urol 167:1243-1247, 2002

21. Moretti MA, Cesar LA, Nusbacher A, Kern KB, Timerman S, Ramires JA: Advanced cardiac life support training improves long-term survival from in-hospital cardiac arrest. Resuscitation 72:458-465, 2007

22. Philibert I, Friedmann P, Williams WT: New requirements for resident duty hours. JAMA 288:1112-1114, 2002

23. Selden NR, Anderson VC, McCartney S, Origitano TC, Burchiel KJ, Barbaro NM: Society of Neurological Surgeons boot camp courses: knowledge retention and relevance of hands-on learning after 6 months of postgraduate year 1 training. J Neurosurg 119:796-802, 2013

24. Selden NR, Barbaro N, Origitano TC, Burchiel KJ: Fundamental skills for entering neurosurgery residents: report of a Pacific region "boot camp" pilot course, 2009. Neurosurgery 68:759-764, 2011

25. Selden NR, Origitano TC, Burchiel KJ, Getch CC, Anderson $\mathrm{VC}, \mathrm{McCartney} \mathrm{S,} \mathrm{et} \mathrm{al:} \mathrm{A} \mathrm{national} \mathrm{fundamentals} \mathrm{curricu-}$ lum for neurosurgery PGY1 residents: the 2010 Society of Neurological Surgeons boot camp courses. Neurosurgery 70:971-981, 2012

26. Seymour NE, Gallagher AG, Roman SA, O'Brien MK, Bansal VK, Andersen DK, et al: Virtual reality training improves operating room performance: results of a randomized, double-blinded study. Ann Surg 236:458-464, 2002

27. Smeets T, Wolf OT, Giesbrecht T, Sijstermans K, Telgen S, Joëls M: Stress selectively and lastingly promotes learning of context-related high arousing information. Psychoneuroendocrinology 34:1152-1161, 2009

28. Sutton RM, Niles D, Meaney PA, Aplenc R, French B, Abella BS, et al: Low-dose, high-frequency CPR training improves skill retention of in-hospital pediatric providers. Pediatrics 128:e145-e151, 2011

29. Wehbe-Janek H, Colbert CY, Govednik-Horny C, White BA, Thomas S, Shabahang M: Residents' perspectives of the value of a simulation curriculum in a general surgery residency program: a multimethod study of stakeholder feedback. Surgery 151:815-821, 2012

\section{Disclosures}

The authors report no conflict of interest concerning the materials or methods used in this study or the findings specified in this paper.

\section{Author Contributions}

Conception and design: Selden. Acquisition of data: all authors. Analysis and interpretation of data: all authors. Drafting the article: all authors. Critically revising the article: Selden, Siler, Cleary. Reviewed submitted version of manuscript: Selden, Siler, Cleary. Approved the final version of the manuscript on behalf of all authors: Selden. Study supervision: Selden.

\section{Correspondence}

Nathan R. Selden, Department of Neurological Surgery, Oregon Health \& Science University, Mail code: CH8N, 3303 SW Bond Ave., Portland, OR 97239. email: seldenn@ohsu.edu. 